\title{
Wodurch setzen wir die Mortalität der Appendicitis herab und verhüten Abszesse. und Peritonitiden?')
}

Von Prof. Hermann Kümmell in Hamburg.

(Fortsetzung aus No. 25.)

Sie sehen, meine Herren, daß wir trotž der erwähnten, in ihrer Bedeutung gewiß nicht zu unterschätzenden Untersuchungsmethoden außerstande sind, in prognostischer Beziehung einen Fortschritt aufzuweisen. Mögen uns die bekannten diagnostischen Hilfsmittel, die einzelnen bisher für charakteristisch gehaltenen Symptome, sowie die eben erwähnten neueren Untersuchungsmethoden in ausgeprägten Fällen über den momentanen Zustand der Bauchhöhle Aufschluß geben, in dem wichtigsten Punkt, über die Beschaffenheit der Appendix, selbst wenn sie schwer erkrankt, lassen sie uns vollkommen im Dunkeln. Die Prognose, der Ausblick über den weiteren Verlauf des einzelnen Falles, darüber, was die nächsten Stunden und der folgende Tag bringt, bleibt vollkommen unaufgeklärt. Das ist das Unbefriedigende, das Traurige und Unvollkommene unseres Könnens, das ist es, was die Appendicitis so unheimlich und gefürchtet erscheinen läßt, daß wir in so vielen Fällen kein Urteil über den Zustand der Appendix haben und noch weniger einen prognostisch annähernd sicheren Ausblick in das weitere Schicksal derselben tun können. Wenigstens ist dies meiner Mitarbeiter und mein subjektives Empfinden, das gewiß jeder von Ihnen, der zahlreiche Operationen im akuten Stadium zu machen Gelegenheit hatte, mit uns teilen wird. Es kommt weit häufiger vor, daß man, gestützt auf die anscheinend leichten klinischen Symptome, eine nur wenig veränderte Appendix, eine Appendicitis simplex zu finden glaubt und statt dessen durch eine gangränöse, zum Platzen gefüllte oder bereits perforierte Appendix bei der Frühoperation überrascht wird. Nur selten fanden wir günstigere Verhältnisse bei der Operation, als wir angenommen hatten.

Woher kommt es denn sonst, daß uns immer noch eine so große Zahl von zirkumskripteu und diffusen Peritonitiden überwiesen wird, woher kommt es, daß eine so ungemein große Anzahl von Abszessen in einem relativ kurzen Zeitraum, wie z. B. 125 auf der Lenhartzschen Abteilung, zur Beobachtung gelangten? Warum müssen noch, wie ich erwähnte, in dem blühendsten Alter zwischen dem 10. und 25. Lebensjahr mehr Menschen an Appendicitis sterben, als an allen übrigen Krankheiten zusammen? Ich

1) Apotheker Hadra, Berlin, Spandauerstr. 77.

2) Vortrag auf dem 39. Kongreß der Deutschen Gesellschaft für Chirurgie 1910. 
habe es oft von hocherfahrenen Praktikern gehört, wenn wir bei einem mit anscheinend geringen klinischen Symptomen ohne Fieber und Pulssteigerung einhergehenden Krankheitsfall bereits eine perforierte Appendix mit allen ihren Folgeerscheinungen vorfanden, daß es eine traurige, aber zurzeit nicht zu ändernde Wahrheit sei, daß wir außerstande sind, mit den früher als zuverlässig angesehenen diagnostischen Merkmalen einen annähernd sicheren Rückschluß auf den Zustand des krankmachenden Organs und auf den weiteren Verlauf zur machen.

Die Wissenschaft und mit ihr die Menschheit muß denjenigen, welche uns bei dem Mangel an diagnostischen und prognostischen Können den einzigen gangbaren Weg gewiesen haben, durch frühzeitiges Eingreifen die Menschen zu heilen und alle traurigen Konsequenzen eines langen Krankenlagers, der AbszeBbildung und der Peritonitiden definitiv zu verhiiten, zu größtem Dank verpflichtet sein. In der Frage der sicheren und positiven Heilung der Appendicitis nützt kein Deliberieren und Diskutieren, keine Sirenenklänge der Statistik da hilft nur ein zielbewußtes und rechtzeitiges Handeln. „Principiis obsta" ist in der Appendicitis-Frage wohl ebenso wichtig wie kaum bei einer anderen Erkrankung. Wir brauchen keine Peritonitis und keine Abszesse bei den Patienten zu beobachten, wir können sie mit Sicherheit vermeiden, wenn wir die Kranken rechtzeitig in Behandlung bekommen.

Warum hört man so oft bei später beobachtetem Krankheitsverlauf, daß die Krankheit so leicht begonnen und so günstig verlaufen sei, bis plötzlich unerwartet eine so wesentliche Verschlimmerung eingetreten. sei? Warum klingt einem so oft das unheimliche ,,zu spät" entgegen, wo man sich sagen muß, das Leben hätte man bei einem rechtzeitigen Vorgehen, wie in gleichen Fällen, wohl retten können?

Es wird wohl ein jeder zugeben müssen, daß wir in der Frühoperation das Mittel besitzen, das uns in den Stand setzt, die an Appendicitis Erkrankten in kürzester Zeit definitiv zu heilen, und zwar mit der geringsten Mortalität. Ein Mittel, das jedes Rezidiv und jede schwere weitere oft so verhängnisvolle Folgekrankheit in der Regel ausschließt. Ehe wir zur Beantwortung der Frage übergehen, ob die Frühoperation auch praktisch durchzuführen ist, möchte ich zunächst meinen Standpunkt in der Frage präzisieren, ob wir unbesehen und kritiklos jeden Fall von Appendicitis operieren sollen; auf diesem radikalen Standpunkt stehe ich nicht.

Es gibt eine ungemein große Anzahl von Appendizitiden so leichter Art, so rasch vorübergehend und von so geringer Intensität, daß bei ihnen eine sofortige Operation nicht nötig ist. Dahin gehört die große Zahl der chronischen Formen, welche immer wieder leichte Exazerbationen zeigen, welche stets an der typischen Stelle den Schmerzpunkt haben und in mehr oder weniger großer Zahl unsere Sprechstunden aufsuchen, welche einige Stunden oder einen Tag der Ruhe pflegen, um dann wieder hergestellt zu sein. Fälle, die oft rezidivieren und immer von neuem mit ihren Beschwerden zum Arzt kommen, ohne daß jemals ein ausgeprägter Anfall auftritt. Diese leichtesten oder ambulanten Fälle von Appendicitis, wie ich sie nennen möchte, habe ich in einer ungemein großen Zahl beobachtet, aber niemals akut operiert, sondern nur, wenn dauernde, den Lebensgenuß und die Arbeitsfähigkeit störende Erscheinungen oder belästigende Rückfälle eintraten. Wissen wir doch, daß so mancher für nervös gehaltene, sich elend fühlende Patient nach Entfernung des nagenden Wurms ein anderer, ein arbeitsfähiger Mensch wurde. Wissen wir doch, daß gerade den Kindern mit ihren häufigen kurzdauernden Schmerzattacken, mit ihrem daniederliegenden Appetit und gestörten Allgemeinbefinden, mit dem charakteristischen, plötzlich eintretenden Erblassen und Verändern ihrer Gesichtszüge mit einem Schlage geholfen wird, daß sie $\mathrm{zu}$ gesunden, frischen Menschenkindern werden, wenn endlich die krankmachende Ursache erkannt und die Appendix beseitigt ist.

Das sind nicht die Fälle, welche zur Operation drängen, oder bei denen im Warten Gefahr liegt und die wir hier in das Bereich unserer heutigen Betrachtung ziehen wollen, das sind die Fälle, die vorzüglich mit Rizinusöl, Opium oder mit einer indifferenten Behandlung heilen und nach Rückfällen mehrfach heilen und den hohen Prozentsatz der guten Statistik der intern behandelten Fälle ausmachen. Von den Hunderten derartiger Fälle, die ich beobachtet und nicht operiert habe, habe ich naturgemäß nicht einen einzigen verloren und eine Mortalität von $0 \%$.

Diese große Gruppe scheidet aus dem Bereich der akuten operativen Behandlung.

Unser aktives Vorgehen ist gegen den akuten Appendicitisanfall gerichtet, welcher in einer Reihe von Stunden ohne selbstverständlich das den Schmerz lindernde und dadurch täuschende Opium nicht zum Abklingen kommt, bei dem mit oder ohne Fieber die Schmerzen weiter fortbestehen, oder der geschilderte kurzdauernde intensive Schmerz einem schmerzlosen Stadium gewichen ist. Ist die Diagnose der akuten Appendicitis gestellt, so sollten wir nicht den weiteren Verlauf dem ungewissen Schicksal überlassen, sondern durch ein aktives Vorgehen eine schnelle und definitive Heilung anbahnen.

Bei dem Streben, einen diagnostischen resp. prognostischen Anhaltspunkt zu gewinnen, um schwere Fälle von leichten zu unterscheiden, hat Herr Sonnenburg das verlassene und gefürchtete Rizinusöl gleichsam als Agent provocateur in die Therapie wieder eingeführt. Ich zweifele nicht, daß eine große Reihe der leichten Formen von Appendicitis von der Gruppe, wie ich sie bereits skizziert habe, durch Ricinusöl geheilt werden, d. h. daß sie sich nach Befreiung des Darmes und des Coecums von stauenden Kotmassen subjektiv wohl fühlen. Ich zweifle aber ebenso wenig, daß bei denselben Patienten derselbe Heileffekt auch ohne Rizinusöl erzielt worden wäre.

Die Gefahr der Rizinusölbehandlung liegt auf einer anderen Seite. Wenn sich Herl Sonnenburg in seiner Mitteilung vorsichtig ausdrückt und erklärt, daß diese Behandlung nur für das Krankenhaus zu empfehlen sei, um beim Fehlschlagen dieser Therapie einen operativen Eingriff folgen zu lassen, so ist es trotzdem zu naheliegend und menschlich sehr verständlich, daß auch der Praktiker außerhalb des Krankenhauses das Rizinusöl zur Anwendung bringt, gestützt auf das Urteil eines erfahrenen Mannes wie Sonnenburg, und dadurch den für den weiteren Verlauf so wichtigen günstigen Zeitpunkt der Operation verstreichen läßt. Darüber müssen wir uns klar sein, daß eine interne Behandlungsmethode, welche der in der Praxis stehende Arzt nicht anwenden darf, keinen hohen Wert besitzen kann; denn der Praktiker ist es, welcher in der überwiegenden Mehrzahl der Fälle die Patienten zuerst sieht, zuerst behandelt und dadurch über ihr weiteres Schicksal definitiv entscheiden kann.

Eine weitere Schattenseite und Gefahr der Rizinusölbehandlung besteht darin, daß sie ebenso wie das Opium ein subjektives Wohlbefinden der Kranken vortäuscht. Wir haben mehrfach Patienten, darunter auch Aerzte in Behandlung bekommen, die gegen ihren anscheinend leichten Appendixanfall abends zuvor Rizinusöl nahmen, sich am andern Tag subjektiv wohler fühlten, sich jedoch, um definitiv und schnell geheilt zu werden, zur Frühoperation entschlossen. In diesen Fällen fanden wir Gangrän der Schleimhaut, einmal sogar eine weit vorgeschrittene Zerstörung der Appendix. Ich sehe ganz ab von den zahlreichen Fällen früherer Jahre, in denen schwere und schwerste Fälle nach Rizinusbehandlung uns in der traurigsten Verfassung überwiesen wurden.

Daß die von Herrn Sonnenberg nach der Darreichung von Rizinusöl als geheilt bezeichneten Patienten wirklich geheilt und nicht nur behandelt sind, dafür muß uns natürlich nach einigen Jahren, nachdem ihre Rezidivfreiheit festgestellt ist, erst der Beweis erbracht werden, denn ich zweifle, daB diese vorübergehenden Heilungen vom akuten Anfall entgegen allen unseren tausendfachen Erfahrungen wirklich Heilungen sind.

Stehen wir nach dem Gesagten noch auf dem Standpunkt, daß wir bei der Unmöglichkeit einer einigermaßen sicheren Prognose über den weiteren Verlauf der diagnostizierten akuten Appendicitis in der Frühoperation das einzig sichere Mittel besitzen, um den Kranken definitiv und schnell zu heilen, ihn 
vor weiteren unberechenbaren Gefahren, der Abszeßbildung, der Peritonitis, der Sepsis u.a.m., mit einer großen Wahrscheinlichkeit zu schützen, so drängt sich uns die weitere Frage auf: ist die Frühoperation auch wirklich praktisch durchzuführen? Die Möglichkeit ihrer mehr oder weniger allgemeinen Durchführung wird von einigen inneren Klinikern bestritten. Ich halte ihre Ausführung für sehr wohl möglich, wenn wir nur wollen, d. h. wenn wir die Unterstützung derjenigen Kollegen finden, welche die Patienten zuerst zu sehen bekommen, und wenn die Patienten und deren Angehörige in der Frage der Appendicitis oder vielmehr über die akuten Beschwerden, welche sich im Bereiche des Darmes oder des Bauches abspielen, weiter aufgeklärt werden, wenn, wie ein Hamburger Kollege sehr richtig sagt, bei dem Publikum an Stelle der Perityphlitisangst eine Peritiyphlitiseinsicht treten wird.

Was zunächst die Schwierigkeit der Frühdiagnose anbetrifft, so habe ich bereits des längeren auseinandergesetzt und oft genug betont, daß die Diagnose der Perityphlitis überhaupt und besonders in den ersten Stunden recht schwierig sein kann, daß es stets Fälle geben wird, in denen auch das Können des Erfahrenen versagt, wo Irrtümer unterlaufen und die wirklich vorhandene Krankheit verkannt wird.

Ich will auch nicht näher auf die allseitig bekannten differentialdiagnostischen. Schwierigkeiten eingehen, auf die Verwechslung der Appendicitis mit den Erkrankungen der nahegelegenen weiblichen Genitalorgane, der Salpingitis und der häufigen Kombination beider Formen, der in ihren Folgen so schweren und immer noch nicht genug bekannten und berücksichtigten Tatsachen, auf welche ich schon in früheren Publikationen wiederholt aufmerksam gemacht habe, daß während der Menstruation eine Verschlimmerung oder ein Rezidivieren der Appendicitis leicht eintritt und daß oft schwere Erscheinungen der Wurmfortsatzerkrankungen als Menstruationsbeschwerden gedeutet werden. So führt z. B. Lenhartz einen bezüglichen, ein 19jähriges Mädchen betreffenden Fall an, welches 24 Stunden vor Erscheinen der Menses mit heftigen, wehenartigen Leibschmerzen und geringem Fieber erkrankt war. Es bestanden nach 36 Stunden kolikartige Schmerzen in Verbindung mit der schußweise erfolgenden Blutentleerung. Die Differentialdiagnose schwankte zwischen einer akuten Appendicitis, einer Peritonitis und schweren nervösen Zuständen. Es wurde abgewartet und erst am anderen Tage bei der operativen Eröffnung reichlich Eiter gefunden. Nach vorübergehender Besserung von. sieben. Tagen trat nach weiteren zehn Tagen der Tod ein; Isenhartz ist im Zweifel, ob nicht durch längeres Abwarten oder durch sofortiges Eröffnen ein günstiger Ausgang hätte erzielt werden können. Für mich ist es, gestützt auf meine Erfolge mit der Frühoperation, außer Zweifel, daß ein frühzeitiger operativer Eingriff aller Wahrscheinlichkeit nach ein erfolgreiches Vorgehen gewesen wäre. Das Unglück war in diesem Falle, daß die kurz vor der Menstruation eingetretene Appendicitis nicht rechtzeitig diagnostiziert war oder vielleicht nicht diagnostiziert werden konnte.

Wenn ich weiterhin an die in den Anfangsstadien so ungemein schwierige Differentialdiagnose zwischen Pneumonie und Appendicitis erinnere, an die Verweschlung mit Nierensteinkoliken oder sonstigen renalen Erkrankungen, besonders wenn eine Miterkrankung dieses Organs (Blut, Albumen u. a.) noch vorhanden ist, weiterhin an die Verwechslung mit Ulcus duodeni oder ventriculi, welch letzteres vielfach als Folgeerscheinung und Komplikation der Appendicitis vorkommt, so glaube ich damit die wichtigsten Punkte angedeutet zu haben.

Die Zahl der Fehldiagnosen, welche durch die diagnostischen und differentialdiagnostischen Schwierigkeiten bedingt sind und eine immerhin recht kleine Zahl von $3,3 \%$, wie man annimmt, darstellt, wird durch die zunehmende Erfahrung des Einzelnen immer geringer werden und prozentarisch kaum in die Wagschale fallen. Hier ist das Gebiet, auf dem durch gemeinsame Arbeit der Chirurgen mit den Internen noch vieles erreicht werden kann, wo durch die gründliche Ausbildung unserer studierenden Jugend auf diesem Gebiete dem zukünftigen Arzt eine weitgehende Erfahrung und vertiefte Kenntnis mit auf den Weg gegeben werden kann.
Die Diagnose der akuten Appendicitis ist vor Ablaufderersten 24.Stundenzustellen. Ausnahmen kommen vor, sind jedoch selten. In derselben Zeit kann sehr wohl festgestellt werden, ob es sich um einen leichten, einen operativen Eingriff nicht erfordernden oder um einen prognostisch zweifelhaften oder gar einen schweren Fall handelt. Dabei sollen wir nicht vergessen, daß die Zahl der mit unzweideutigen oder gar schweren Symptomen einsetzenden Fälle recht groß ist. - Diesen Zeitraum von 24 Stunden möchte ich als die äußerste Zeitgrenze ansehen, in der der Würfel gefallen sein muß. Entweder ist der Anfall vorüber, oder bei noch bestehenden Krankheitserscheinungen oder prognostischen Zweifeln ist die Operation auszuführen. Bei den Fällen mit zweifelloser Diagnose ist die Operation sofort auszuführen. Dank der Einsicht einer großen Anzahl vorurteilsfreier Kollegen sind uns die Patienten mehr und mehr im frühen, ja im frühsten Stadium, wenige Stunden nach Beginn des Anfalls zugeführt und geheilt. Auch in diesen Fällen fanden wir vielfach schon die Appendix brandig und perforiert mit Exsudat in der Bauchhöhle vor.

Bei diesem Vorgehen haben wir in den letzten drei Jahren von 347 innerhalb der ersten 48 Stunden Operierten überhaupt nur $2-0,5 \%$ verloren. Wie Sie aus der beigefügten Tabelle I ersehen, 1907 keinen, 1908 unter 120 und 1909 unter 143 je einen. Die Mortalität bei der Frühoperation ist innerhalb der ersten 50 Stunden, abgesehen von dem Jahre 1903, in welchem wir sie allmählich einführten und unter fünf Operierten einen verloren hatten, von $8 \%$ i m Jahre 1904 a uf 7,5 des Jahres 1905 und $3,4 \%$ des Jahres 1906 bis zu dem erwähnten günstigen Prozentsatz der drei letzten Jahre, $1907=$ $0 \%, 1908=0,8 \%$ und $1909=0,6 \%$ gesunken.

DieGesamtmortalität der frühoperierten 574 Fälle innerhalb der ersten 48 Stunden beträgt $12=2 \%$.

Nehmen wir dazu die 1595 Intervalloperationen Tabelle 3, welche im letzten und im laufenden Jahr keinen und 1908 nur einen Todesfall aufzuweisen hatten, mit einer Gesamtmortalität von 15 Fällen $=1 \%$ und stellen diese unsern sämtlichen wegen Appendicitis ausgeführten Operationen gegenüber, so ergibt sich ein auffallendes Mißverhältnis.

Tabelle 2 .

Operationen im akuten Stadium.

\begin{tabular}{c||c|c|c|c|c}
\hline \hline Jahr & Anzahl & $\begin{array}{c}\text { Operation } \\
\text { inden ersten } \\
\text { 24 Stunden }\end{array}$ & gesund & tot & $\begin{array}{c}\text { Mortalität } \\
\text { in Prozenten }\end{array}$ \\
\hline \hline 1903 & 5 & 0 & 5 & 0 & 0 \\
1904 & 37 & 17 & 34 & 3 & 8,0 \\
1905 & 53 & 23 & 49 & 4 & 7,5 \\
1906 & 88 & 49 & 85 & 3 & 3,4 \\
1907 & 84 & 61 & 84 & 0 & 0 \\
1908 & 120 & 62 & 119 & 1 & 0,8 \\
1909 & 143 & 89 & 142 & 1 & 0,6 \\
1910 & 44 & 5 & 44 & 0 & 2 \\
\hline Summa & 574 & & 562 & 12 & 2
\end{tabular}

Tabelle 3 .

Gesamtzahl der Intervalloperationen.

\begin{tabular}{c||r|r|r|l}
\hline \hline Jahr & Zahl & gesund & tot & $\begin{array}{c}\text { Mortalitä } \\
\text { in Prozenten }\end{array}$ \\
\hline \hline 1889 & 4 & 4 & 0 & 0 \\
1890 & 0 & 0 & 0 & 0 \\
$\mathbf{1 8 9 1}$ & 3 & 3 & 0 & 0 \\
1892 & 6 & 6 & 0 & 0 \\
1893 & 5 & 5 & 0 & 0 \\
1894 & 20 & 20 & 0 & 0 \\
1895 & 18 & 18 & 0 & 0 \\
1896 & 22 & 22 & 0 & 0 \\
1897 & 20 & 20 & 0 & 0 \\
1898 & 40 & 40 & 0 & 0 \\
1899 & 42 & 41 & 1 & 2,3 \\
1900 & 60 & 57 & 3 & 5,0 \\
1901 & 54 & 53 & 1 & 1,8 \\
$\mathbf{1 9 0 2}$ & 71 & 71 & 0 & 0 \\
1903 & 84 & 83 & 1 & 1,2 \\
1904 & 129 & 128 & 1 & 0,7 \\
1905 & 187 & 185 & 2 & 1,0 \\
1906 & 161 & 159 & 2 & 1,2 \\
1908 & 188 & 185 & 3 & 1,5 \\
1909 & 217 & 216 & 1 & 0,4 \\
1910 & 224 & 224 & 0 & 0 \\
\hline Summa & 40 & 40 & 0 & 0 \\
\hline
\end{tabular}

Tabelle 4 gibt uns einen Ueberblick über die sä $\mathrm{m}$ tlichen Appendixoperationen, welche wir im Laufe der Jahre auf 
meiner Abteilung und außerhalb des Krankenhauses ausgeführt haben. Die Gesamtzahl beträgt 2651 mit 181 Todesfällen $=6,8 \%$.

Sie sehen aus dieser Zusammenstellung, wie die Gesamtmortalität nach der weiteren Ausgestaltung der Frühoperation nach dem Jahre 1904 und besonders nach Einführung der Rehnschen Methode der Peritonitisbehandlung wesentlich gesunken ist, von 12,9 auf 6,2 im Jahre 1905 und weiterhin auf 3,9 im Jahre 1908, 3,5 im Jahre 1909 und 2,2 im ersten Quartal des laufenden Jahres. Diese Statistik hat deshalb wohl einen gewissen Wert, weil sie sich auf ein nach einheitlichen Gesichtspunkten hin von demselben Autor behandeltes Krankenmaterial bezieht, was bei einer Sammelstatistik nicht der Fall ist.

An der immerhin hohen Gesamtmortalität von 6,8 oder von $3,5 \%$ im günstigsten Jahre 1909 gegenüber einer Mortalität von $0,6 \%$ für die Frühoperationen und $0 \%$ für die Intervalloperationen desselben Jahres tragen die Schuld die Spätoperationen, die Peritonitiden und Abszesse, also die Fälle, welche zu spät operiert sind und durch rechtzeitige Operation zum größten Teil hätten gerettet werden können.

Tabelle 4.

Gesamtzahl der Appendicitisoperationen.

\begin{tabular}{c||c|c|c|c}
\hline \hline Jahr & Zahl & gesund & tot & $\begin{array}{c}\text { Mortalität } \\
\text { in Prozenten }\end{array}$ \\
\hline \hline 1888 & 1 & 1 & 0 & 0 \\
1889 & 7 & 7 & 0 & 0 \\
1890 & 1 & 0 & 1 & + \\
1891 & 3 & 3 & 0 & 0 \\
1892 & 7 & 7 & 0 & 0 \\
1893 & 9 & 7 & 2 & 22,2 \\
1894 & 23 & 22 & 1 & 4,3 \\
1895 & 19 & 18 & 1 & 5,3 \\
1896 & 38 & 33 & 5 & 13,1 \\
1897 & 33 & 29 & 4 & 12,1 \\
1898 & 49 & 46 & 3 & 6,1 \\
1899 & 55 & 48 & 7 & 12,7 \\
1900 & 78 & 67 & 11 & 14,8 \\
1901 & 75 & 66 & 9 & 12,0 \\
1902 & 103 & 93 & 10 & 9,7 \\
1903 & 139 & 124 & 15 & 10,7 \\
1904 & 224 & 195 & 29 & 12,9 \\
1905 & 274 & 257 & 17 & 6,2 \\
1906 & 380 & 266 & 14 & 5,0 \\
1907 & 306 & 287 & 19 & 6,2 \\
1908 & 403 & 392 & 16 & 3,9 \\
1909 & 423 & 408 & 15 & 3.5 \\
1910 & 96 & 94 & 2 & 2,2 \\
\hline Summan & 2651 & 2470 & 181 & 6,8 \\
\hline
\end{tabular}

In der Gruppe Abzesse habe ich die zirkumskripten Peritonitiden, die abgekapselten und multiplen Abszesse, welche als Spätoperationen nach 48-50 Stunden, vom dritten Tage ab zur Operation gelangten, zusammengefaßt, unter Peritonitis ausschließlich die diffusen Peritonitiden, die die ganze Bauchhöhle einnehmenden eitrigen und jauchigen Prozesse.

Bei den Peritonitiden müssen wir außerdem berücksichtigen, daß sie sehr oft in einem recht traurigen und späten Stadium in unsere Behandlung gelangten. Wenn beispielsweise in der Lenhartzschen Tabelle ${ }^{1}$ ) 13 Fälle von akuter Perforationsperitonitis angeführt werden, welche nach dem neuen Verfahren operiert wurden, mit fünf Todesfällen, so sei dazu bemerkt, daß vier dieser Fälle trotz gestellter Diagnose einer diffusen Peritonitis mehrere Tage auf der Inneren Abteilung konservativ behandelt wurden. Es ist leicht verständlich, daß solche Fälle die interne Statistik entlasten und die chirurgische Mortalitätsstatistik schwer belasten.

(Schluß folgt.) 